\title{
Cross-kingdom metabolic manipulation promotes Salmonella replication inside macrophages
}

\author{
Deyanira Pérez-Morales (i) ${ }^{1} \&$ Víctor H. Bustamante (i] ${ }^{1 凶}$
}

Replication inside macrophages is crucial for systemic dissemination of Salmonella in hosts. In a Nature Communications article, Jiang et al. show that Salmonella stimulates glycolysis and represses serine synthesis in macrophages, leading to accumulation of host glycolytic intermediates that the bacteria use as carbon source and as cues for its replication.

\section{Salmonella pathogenicity and SopE2}

Salmonella enterica serovar Typhimurium (here referred as Salmonella) counts with multiple factors and mechanisms to infect humans and other animals ${ }^{1}$. Its pathogenicity was mainly shaped by the acquisition of foreign DNA through multiple horizontal transfer events. In particular, Salmonella pathogenicity islands 1 and 2 (SPI-1 and SPI-2) are two gene clusters that were acquired by Salmonella at different evolutionary times ${ }^{2}$, each one encoding a Type III Secretion System (T3SS) together with different effector proteins and transcriptional regulators ${ }^{1}$.

A T3SS is a multiprotein complex similar to a syringe, through which bacteria inject effector proteins directly into the cytoplasm of host cells ${ }^{3}$. Effectors injected by the SPI-1 T3SS, including the guanine nucleotide exchange factor (GEF) SopE2, induce cytoskeletal rearrangements and activate signaling cascades that lead to internalization of Salmonella into the intestinal epithelium cells, causing enteritis ${ }^{1}$. The resulting intestinal inflammation mediated by SPI-1 effectors, and to some extent also by SPI-2 effectors, generates diverse antimicrobial responses and production of specific compounds that support the growth of Salmonella, but not of commensal bacteria, helping the pathogen to outcompete the intestinal microbiota ${ }^{4}$.

The SPI-2 T3SS and its effectors enable the bacteria to replicate inside macrophages ${ }^{1,5}$. In particular, SPI-2 effectors modulate diverse cellular processes to establish the Salmonella containing vacuole (SCV), a niche for Salmonella replication within macrophages that facilitates evasion of antibacterial responses and efficient acquisition of nutrients ${ }^{5,6}$. Some SPI-1 effectors, including SopE2, also contribute to SCV biogenesis and intracellular replication by unclear mechanisms ${ }^{7}$.

\section{Salmonella induces metabolic alterations in macrophages}

A recent Nature Communications paper by Jiang et $\mathrm{al}^{8}$. uncovers fascinating new mechanisms mediating Salmonella virulence, involving the direct manipulation of macrophage metabolism through effector SopE2 (Fig. 1). Jiang et al. show that Salmonella stimulates aerobic glycolysis in macrophages, while the tricarboxylic acid (TCA) cycle and oxidative phosphorylation activities are reduced ${ }^{8}$. This metabolic reprogramming resembles that occurring in proliferating cancer

\footnotetext{
${ }^{1}$ Departamento de Microbiología Molecular, Instituto de Biotecnología, Universidad Nacional Autónoma de México, Cuernavaca, Morelos, México.

凶email: victor.bustamante@ibt.unam.mx
} 


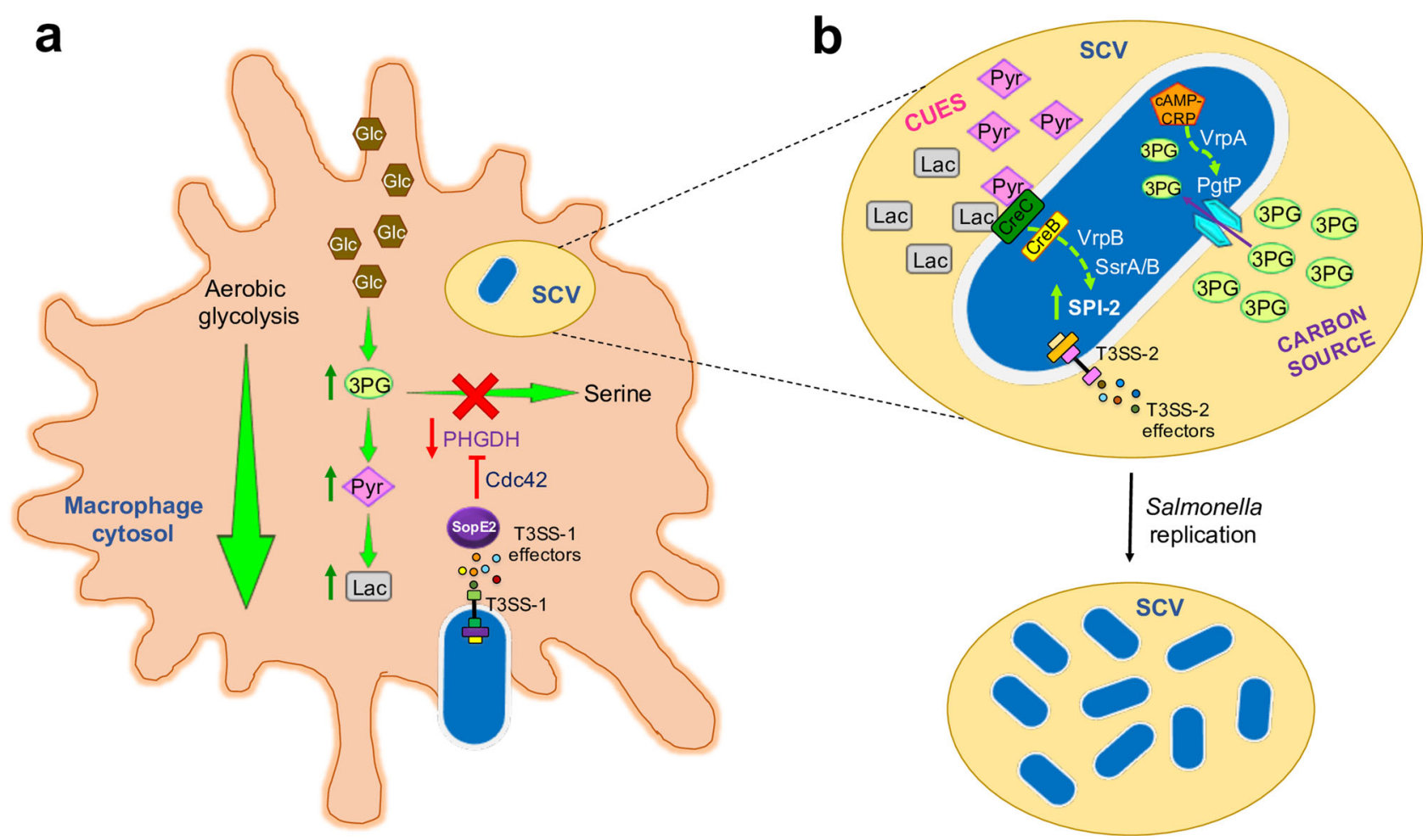

Fig. 1 Salmonella manipulates macrophage metabolism, thus obtaining a carbon source and cues for intracellular replication. a Following infection with Salmonella, macrophages increase the conversion of glucose (Glc) to lactate (Lac) through aerobic glycolysis. Additionally, the SopE2 effector, which is injected by Salmonella into the cytoplasm of host cells by the SPI-1 T3SS (T3SS-1), represses serine synthesis by inhibiting expression of PHGDH via the host cell Rho GTPase Cdc42. Increased aerobic glycolytic flux and reduction of serine synthesis prompt the accumulation of host 3-phosphoglycerate (3PG), Lac, and pyruvate (Pyr). b Within the Salmonella containing vacuole (SCV), bacterial replication is supported by the use of host 3PG as a carbon source, while host Lac and Pyr stimulate expression of SPI-2 genes (including those encoding T3SS-2 and effector proteins). In bacteria, the cAMP-CRP complex senses low levels of host Glc and activates the expression of PgtP (3PG transporter) through the VrpA transcriptional regulator. Furthermore, the two-component system $\mathrm{CreB} / \mathrm{C}$ senses host Lac and Pyr and activates the expression of the VrpB transcriptional regulator, which in turn induces expression of the SsrA/B two-component system, the central positive regulator for SPI-2.

cells, known as the Warburg effect, which transforms most of the incoming glucose to lactate, even under oxygen-rich conditions 9 . It has been proposed that the Warburg effect provides specific nutrients for the multiplication of intracellular bacteria and cancer cells9. Indeed, Jiang et al. report that genetic or biochemical inhibition of macrophage glycolysis reduces intracellular replication of Salmonella ${ }^{8}$.

Interestingly, the authors also reveal that Salmonella-infected macrophages exhibit reduced serine synthesis and diminished activity of downstream metabolic pathways (glycine and glutathione synthesis) ${ }^{8}$. They show that the GEF SopE2 effector and its host target, Rho GTPase Cdc42, inhibit macrophage serine synthesis via downregulation of the phgdh gene, encoding a key enzyme in the pathway. Consistently, Jiang et al. report that SopE2 is necessary for effective systemic infection of mice by Salmonella, and that both SopE2 and Cdc42 are important for intracellular replication of the bacteria ${ }^{8}$.

The precise mechanisms by which Salmonella induces metabolic reprogramming in macrophages, particularly the link between SopE2-Cdc42 and phgdh expression, remain yet to be determined. It will also be interesting to understand the potential effects of such metabolic reprogramming on macrophage antimicrobial responses.

\section{Host metabolites act as nutrients and as signals for the pathogen}

Jiang et al. show that the Salmonella-induced effects on macrophage metabolism lead to accumulation of host glycolytic intermediates including 3-phosphoglycerate (3PG), a serine synthesis precursor, which Salmonella uses as carbon source for replication inside macrophages and during infection of mice ${ }^{8}$.

In addition, the low levels of glucose present in infected macrophages induce upregulation of the bacterial 3PG transporter PgtP, through a regulatory cascade involving the cAMP-CRP complex and a previously uncharacterized transcriptional regulator, $\operatorname{VrpA}^{8}$. Thus, the alterations in macrophage metabolism not only provide Salmonella with a carbon source (3PG) but also with a cue (low glucose) that triggers the uptake of 3PG for intracellular replication.

The authors show that increased glycolysis within macrophages leads to accumulation of pyruvate and lactate, which also promote intracellular replication of Salmonella by stimulating SPI-2 gene expression ${ }^{8}$. The mechanism for this upregulation of SPI-2 is mediated by the two-component system CreB/C (known to be activated by pyruvate, lactate, and other short-chain carbon sources $\left.{ }^{10}\right)$, together with a previously uncharacterized regulator (VrpB) and the SsrA/B two-component system (a known positive regulator of $\left.\mathrm{SPI}-2^{1}\right)^{8}$.

Jiang et al. also report succinate accumulation within infected macrophages ${ }^{8}$. Interestingly, a recent study has shown that increased succinate levels in Salmonella-infected macrophages induce expression of SPI-2 genes and of genes associated with antimicrobial resistance ${ }^{11}$. We wonder whether succinate might activate SPI-2 expression through the $\mathrm{CreB} / \mathrm{C}-\mathrm{VrpB}$ regulatory cascade described by Jiang et $\mathrm{al}^{8}$. Interestingly, it is known that Salmonella utilizes microbiota-derived succinate and 
host-derived lactate as carbon sources to efficiently colonize the gut $^{12,13}$.

All these findings suggest that Salmonella, and probably other bacteria, reprogram their own metabolism and regulatory mechanisms to take the best advantage of metabolites present in different niches of their hosts, using them as nutrients and/ or cues.

\section{Concluding remarks}

The study by Jiang et $\mathrm{al}^{8}$. reveals novel mechanisms mediating Salmonella pathogenesis and illustrates some remarkable strategies developed by bacteria to adapt and survive in their hosts (Fig. 1). Other intracellular pathogenic bacteria such as Brucella abortus, Chlamydia pneumoniae, Chlamydia trachomatis, Legionella pneumophila, and Mycobacterium tuberculosis are also known to shift their host cells towards a Warburg-like metabolism (aerobic glycolysis) ${ }^{9}$. This metabolic reprogramming of host cells seems to be pathogen-specific ${ }^{9}$, but the mechanisms behind it are still poorly understood. We expect that future research in this area will provide new strategies to develop anti-infective therapies.

Received: 23 February 2021; Accepted: 3 March 2021;

Published online: 25 March 2021

\section{References}

1. Fàbrega, A. \& Vila, J. Salmonella enterica serovar Typhimurium skills to succeed in the host: virulence and regulation. Clin. Microbiol. Rev. 26, 308-341 (2013).

2. Porwollik, S. \& McClelland, M. Lateral gene transfer in Salmonella. Microbes Infect. 5, 977-989 (2003).

3. Deng, W. et al. Assembly, structure, function and regulation of type III secretion systems. Nat. Rev. Microbiol. 15, 323-337 (2017).

4. Anderson, C. J. \& Kendall, M. M. Salmonella enterica serovar Typhimurium strategies for host adaptation. Front. Microbiol. 8, 1983 (2017).

5. Jennings, E., Thurston, T. L. M. \& Holden, D. W. Salmonella SPI-2 type III secretion system effectors: molecular mechanisms and physiological consequences. Cell Host Microbe 22, 217-231 (2017).

6. Liss, V. et al. Salmonella enterica remodels the host cell endosomal system for efficient intravacuolar nutrition. Cell Host Microbe 21, 390-402 (2017).

7. Vonaesch, P. et al. The Salmonella Typhimurium effector protein SopE transiently localizes to the early SCV and contributes to intracellular replication. Cell Microbiol. 16, 1723-1735 (2014).

8. Jiang, L. et al. Salmonella Typhimurium reprograms macrophage metabolism via T3SS effector SopE2 to promote intracellular replication and virulence. Nat. Commun. 12, 879 (2021).
9. Escoll, P. \& Buchrieser, C. Metabolic reprogramming of host cells upon bacterial infection: Why shift to a Warburg-like metabolism? FEBS J. 285, 2146-2160 (2018).

10. Cariss, S. J., Tayler, A. E. \& Avison, M. B. Defining the growth conditions and promoter-proximal DNA sequences required for activation of gene expression by CreBC in Escherichia coli. J. Bacteriol. 190, 3930-3939 (2008).

11. Rosenberg, G. et al. Host succinate is an activation signal for Salmonella virulence during intracellular infection. Science 371, 400-405 (2021).

12. Spiga, L. et al. An oxidative central metabolism enables Salmonella to utilize microbiota-derived succinate. Cell Host Microbe 22, 291-301 e6 (2017).

13. Gillis, C. C. et al. Dysbiosis-associated change in host metabolism generates lactate to support Salmonella growth. Cell Host Microbe 23, 570 (2018).

\section{Acknowledgements}

Our research is funded by grants from Consejo Nacional de Ciencia y Tecnología (CONACYT)/México (254531) and from Dirección General de Asuntos del Personal Académico de la UNAM (DGAPA)/México (IN206321) to V.H.B. We thank Yvonne Rosenstein by critical reading and editing of the manuscript.

\section{Author contributions}

D.P-M. and V.H.B. contributed to the design and writing of this paper.

\section{Competing interests}

The authors declare no competing interests.

\section{Additional information}

Correspondence and requests for materials should be addressed to V.H.B.

Reprints and permission information is available at http://www.nature.com/reprints

Publisher's note Springer Nature remains neutral with regard to jurisdictional claims in published maps and institutional affiliations.

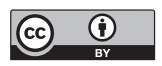

Open Access This article is licensed under a Creative Commons Attribution 4.0 International License, which permits use, sharing, adaptation, distribution and reproduction in any medium or format, as long as you give appropriate credit to the original author(s) and the source, provide a link to the Creative Commons license, and indicate if changes were made. The images or other third party material in this article are included in the article's Creative Commons license, unless indicated otherwise in a credit line to the material. If material is not included in the article's Creative Commons license and your intended use is not permitted by statutory regulation or exceeds the permitted use, you will need to obtain permission directly from the copyright holder. To view a copy of this license, visit http://creativecommons.org/ licenses/by/4.0/.

(C) The Author(s) 2021 